\title{
ÉTICA DESDE LA RESISTENCIA: EL COMPROMISO POLÍTICO DE VICENTE AGUILERA CERNI
}

\author{
Lydia Frasquet Bellver \\ Universitat de Valencia \\ lyfras@gmail.com
}

\begin{abstract}
Este artículo se acerca al trabajo del crítico de arte español Vicente Aguilera Cerni (Valencia, 1920-2005), y analiza su compromiso político con el pensamiento de izquierda a lo largo de su vida.

Palabras clave: Vicente Aguilera Cerni; arte y política; crítica de arte; franquismo; ideología.
\end{abstract}

\section{ETHICS FROM RESISTANCE: THE POLITICAL COMMITMENT OF VICENTE AGUILERA CERNI}

This article approaches the writings of the Spanish art critic Vicente Aguilera Cerni (Valencia, 1920-2005), and analyzes his political commitment to leftist thinking throughout his lifetime.

Key words: Vicente Aguilera Cerni; art \& politics; art criticism; the Franco period; ideology.

Aunque la vida pública de Vicente Aguilera Cerni como político sólo abarca el corto periodo de tiempo en la presidencia del Partido Socialista Popular del País Valenciano (PSP-PV) entre finales de 1975 y hasta la unificación con el PSOE en 1978, su trayectoria de compromiso viene de muy atrás y será un rasgo característico a lo largo de su vida. De manera que no nos ceñiremos a esos años en los que desempeñó oficialmente esta responsabilidad explícitamente orgánica, sino que abordaremos una visión panorámica de su compromiso ideológico a lo largo de toda su existencia. Este objetivo no nos permite sopesar -ya que sería materia para otro estudio- en qué medida reflejó este compromiso en sus escritos.

Escribir no es nunca una tarea inocente, ni tampoco el hecho de seleccionar artistas, reunirlos, apoyarlos profesionalmente; en la vida de Aguilera veremos que, además de esta labor, su compromiso en la denuncia de la realidad opresora del régimen franquista es constante, y lo que va variando es el grado de su visibilidad pública.

Con este artículo queremos evidenciar, a través de los datos de su trayectoria personal, principalmente dos asuntos: calibrar la vinculación de Aguilera con el Partido Comunista de España, y demostrar hasta qué punto el gobierno franquista era conocedor de sus implicaciones políticas e intentó acallarlo. Bien es verdad que una vez llegado el momento de la legalización de los partidos, Aguilera no optó por la vía comunista sino por la propuesta de Tierno Galván. Finalmente analizaremos su actividad en el PSP y su compromiso socialista posterior, que mantuvo hasta el final de sus días. 
Vicente Aguilera Cerni nace en Valencia en 1920, en el seno de una familia acomodada; es hijo de médico militar y nieto por parte de su madre del alcalde de Ceuta, Ricardo Cerni González. Varias personas de su entorno familiar desarrollan sólidas carreras militares, leales en su gran mayoría al bando sublevado, y también su suegro pertenece a este estamento. Sin embargo, desde muy temprano la ideología del joven Aguilera se revela muy diferente a la del núcleo familiar, y se siente más identificado con los ideales progresistas, gracias a que es un lector voraz y, que pese a su juventud, lee todo aquello que está a su alcance.

Ya siendo muy joven, con 16 años frecuenta las filas de la FUE (Federación Universitaria Escolar), la organización de la izquierda estudiantil. Una vez estalla la guerra, en 1937 milita en las Juventudes Socialistas Unificadas (JSU), la organización fundada en 1936 como resultado de la unión entre las juventudes comunistas y socialistas, bajo la dirección de Santiago Carrillo como secretario general. Se suma como voluntario respondiendo a la convocatoria de las JSU y desempeña labores para varios periódicos y publicaciones del frente republicano.

Tras el conflicto bélico regresa a Valencia, donde su madre lo recibe sin rencillas pero no así el resto de la familia, que lo trata con bastante prevención. Ingresa en la universidad, sin conseguir rematar sus estudios ya que cae enfermo; durante una larga convalecencia leerá obsesivamente. En 1941, con 21 años, comienza a escribir sin descanso, tanto poemas como incluso novelas. Fruto de los horrores vividos en el frente, compondrá versos como estos: "Morir, camaradas, es horrible. / Pero así como la noche sabe que detrás llega un mañana, / nosotros sabemos que la noche sigue, que la mañana sólo es del que vive"1.

Al principio de la posguerra Aguilera mantiene su vinculación con el PCE clandestino, trabajando con compañeros como el doctor José Bartrina Villanueva, médico urólogo detenido en 1948 y fallecido en la prisión central de Burgos en 1956 $6^{2}$ Junto a Bartrina, Aguilera se implica en la edición del periódico Verdad, el órgano del Comité Provincial del PCE de Valencia, asumiendo el riesgo de almacenar el periódico en su propio domicilio ${ }^{3}$.

Además, en 1946 entra en contacto con una organización clandestina desarticulada en Valencia, la Unión de Intelectuales Libres. Al parecer, colaboró en organizaciones reiteradamente intervenidas por la policía, quedando desconectado del grupo principal ${ }^{4}$.

Tras estos inicios se produce un paréntesis en su implicación política, o al menos no encontramos actividad filocomunista contrastada durante la década siguiente, la de los años cincuenta. En cambio, será al principio de esa década cuando Vicente Aguilera inicie su desarrollo profesional -al margen de las colaboraciones en guerra que hemos comentado-al publicar artículos en revistas especializadas y en prensa desde 1953. Además Aguilera se vincula laboralmente en 1954 con el Servicio de Estudios Artísticos de la Institución Alfonso el Magnánimo de la Diputación de Valencia, unidad que estaba designada como sección del Instituto Diego de Velázquez del CSIC. Al frente de este servicio de investigación de temas artísticos locales se encuentra como director Felipe $\mathrm{M}^{\mathrm{a}}$ Garín Ortiz de Taranco, cuyos cargos políticos y responsabilidades lo erigen como pieza clave para el régimen en lo concerniente a la organización de las instituciones artísticas valencianas. Seguramente no es casual el abandono de Aguilera de la colaboración comunista en estos años en los que se encuentra trabajando en una institución pública.

\footnotetext{
${ }^{1}$ Aguilera, 2010: 42.

2 Podemos leer sobre el doctor Bartrina en Mancebo, 1988: 242-245.

${ }^{3}$ Marí, 1978: 36-37. El periódico Verdad comenzó a publicarse en 1936 como órgano socialista y comunista; posteriormente en 1968 iniciará su segunda época y numeración desde el no 1 , bajo el título de Verdad, órgano del Comité Provincial de Valencia del PC de España.

${ }^{4}$ Informe de Ministerio de Información y Turismo - oficina de enlace, 7 de marzo de 1967, Archivo General de la Administración, Alcalá de Henares (AGA), Cultura, caja 42/8820.
} 
Recordemos que este periodo temporal se enmarca en el contexto de la Guerra Fría entre los bloques capitalista y comunista, en la que se generó un choque entre dos ideologías fuertemente contrapuestas. El enfrentamiento se filtró a todos los terrenos, desde el político, al militar, intelectual, científico, tecnológico, cultural, e incluso deportivo. Una de las consecuencias fue el desarrollo de una competición cultural entre ambas potencias sin precedentes, marcada por la ideología de cada bloque. Esta batalla ideológica se reflejó en la trayectoria de Vicente Aguilera, ya que de manera contradictoria con su anterior filiación, en esta década de los años cincuenta encontramos a Aguilera vinculado con las actividades promovidas por el gobierno norteamericano. Ese mismo año 1954, ya está participando con la Casa Americana de Valencia, desarrollando una actividad intensa, una colaboración que durará hasta aproximadamente 1960. Siguiendo los rastros de sus intervenciones, no nos queda duda de que el gobierno americano se fijó en Aguilera para servir de altavoz de los valores de su nación en España. ${ }^{5}$

Pese a que el restablecimiento de las relaciones diplomáticas entre España y Estados Unidos data de 1951, y a que el Pacto de Madrid que posibilita el acuerdo económico y militar no se firma hasta 1953, el gobierno americano venía desarrollando actividades culturales en suelo español desde algunos años atrás. Estados Unidos había puesto en marcha la consolidación de un programa cultural principalmente a través de medios de comunicación escritos, así como de la radio y la industria cinematográfica, y del ejercicio de la diplomacia cultural mediante la apertura de centros culturales, las denominadas Casas Americanas, la primera de las cuales, data de 1942 en Madrid. ${ }^{6}$ Estos centros ofrecían exposiciones, conferencias, recitales, bibliotecas bien equipadas y la enseñanza de la lengua inglesa. También se puso en marcha un ambicioso programa educativo que buscaba potenciar el intercambio de profesores y estudiantes de diferentes países, el programa Fullbright, ideado en 1946. Y por último, existió un programa de intercambio informativo y educativo, denominado Foreingn Leaders Program, -para el que Vicente Aguilera estuvo nominado en 1958, pero finalmente no fue seleccionado- destinado a personas cuyo perfil y relieve las convertía en muy significativas para difundir las bondades del país americano. ${ }^{7}$ Todo este esfuerzo intentaba paliar y limpiar la imagen de América en el exterior, y dar a conocer su papel de adalid de la democracia en el mundo.

La relación de Vicente Aguilera Cerni con la Casa Americana de Valencia y con el Centro de Estudios Norteamericanos de Valencia va a ser muy fecunda a partir de 1954 y durante el resto de esa década, y no sólo colabora con esta institución en Valencia, sino que además tendrá presencia activa en el mismo centro en otras provincias, ganará premios convocados por estas entidades, publicará en sus medios y revistas, e incluso formará parte de su organigrama. Sabemos que en 1957 y los años sucesivos Aguilera es Secretario General del Centro de Estudios Norteamericanos de Valencia. No hemos de olvidar tampoco que Vicente Aguilera publicará dos de sus tres primeros libros sobre arte americano -Introducción a la pintura norteamericana (1955) y Arte norteamericano del siglo $X X$ (1957)-, de los cuales el germen indudablemente se encuentra en la labor desarrollada al amparo de esta casa y gracias a los medios que encontró a su disposición en sus instituciones $^{8}$ (Fig. 1).

\footnotetext{
${ }^{5}$ Estas conclusiones las extraigo de mi tesis actualmente en curso, titulada "El historiador y crítico Vicente Aguilera Cerni y el arte español contemporáneo", inscrita en el Departamento de Historia del Arte de la Universitat de València y dirigida por el profesor José Martín Martínez.

${ }^{6}$ El investigador que ha analizado estas vías en profundidad es León, 2009: 133-158, y también Delgado, 2009: 97-132.

${ }^{7}$ Delgado, 2012: 275.

${ }^{8}$ Ambas publicaciones y su contexto han sido recientemente analizados por Bernárdez, 2010: 127-149.
} 
Así pues nos encontramos ante un joven Aguilera que, pese haber publicado poco hasta este momento, tiene el suficiente eco y potencial como para que el Servicio de Información de los Estados Unidos (United States Information Service, USIS) establezca con él este diálogo basado en la modernidad de sus ideas, para promover su participación en coloquios, colaboraciones y conferencias. Se le concede el premio Pi Suñer en 1957 del Instituto de Estudios Norteamericanos de Barcelona por su artículo a la mejor labor cultural sobre temas norteamericanos, en concreto por un artículo sobre Frank Lloyd Wright.

El USIS, desprendemos de este hecho, consideró a Vicente Aguilera Cerni como persona influyente en el campo del arte, capaz de generar una opinión positiva que fortaleciera la imagen de esta nación a nivel cultural. $\mathrm{O}$ dicho de otro modo, de ser seducido para después seducir a los que le rodearon con los conocimientos sobre los valores de la vida y la cultura norteameri-

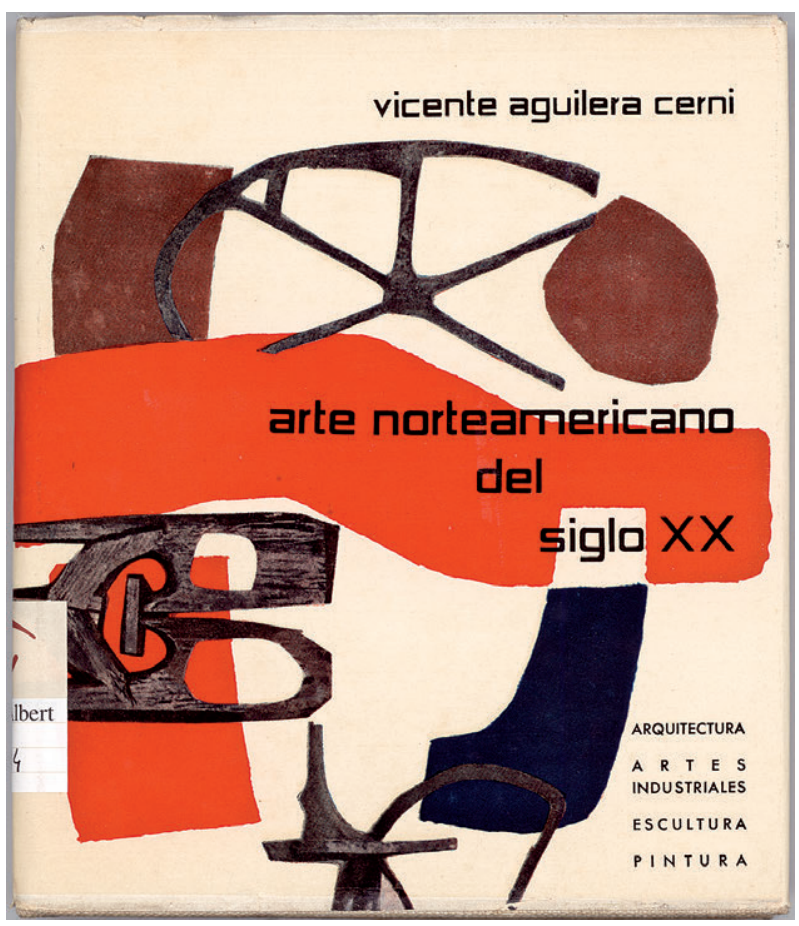

Fig. 1. Portada de Arte norteamericano del siglo XX, 1957. Biblioteca Valenciana Nicolau Primitiu. Biblioteca Gil-Albert. canas. Desconocemos si el USIS tenía noticia de los antecedentes filocomunistas de Vicente Aguilera, pero en definitiva, la ausencia de actividad política por su parte en esta década y su vinculación a una entidad pública, como era la Institución Alfonso el Magnánimo, facilitó que se estableciese esta colaboración mutua durante esos años.

A la vez, el contexto español de posguerra que vivió Aguilera ofrecía muy pocas oportunidades para que un joven con aspiraciones e inteligencia saliera adelante. Sin posibilidad de ejercer en la universidad o en museos, Aguilera se fija en esta organización cultural que publica, da becas, paga artículos, viajes, congresos, y que era una de las pocas vías de ascenso para los intelectuales del momento. Vicente Aguilera encuentra en esta estructura una manera de ver colmada su expectativa y poder publicar.

Será también a partir de esta etapa cuando se sucederán los éxitos internacionales y la publicación de libros, revistas y el trabajo directo con formaciones artísticas: publicará Arte vivo, la revista del Grupo Parpalló -al que dio respaldo teórico- desde 1956 a 1961; se le otorgará el Primer Premio Internacional de la Crítica en la XXIX Bienal de Venecia en 1959, que le dará dimensión internacional a su trabajo, y a partir del cual se sucederán importantes encargos y compromisos profesionales 9 .

Tras esta etapa de vinculación con el Servicio de Información de los Estados Unidos (USIS) en los años cincuenta, volvemos a tener constancia del acercamiento de Aguilera al PCE clandes-

${ }^{9}$ A continuación llegarían para Aguilera otros galardones internacionales, como la medalla especial de la Accademia dei 500 de Roma en 1962, y la medalla de oro de la Presidencia del Consejo de Ministros de Italia en 1965, por los méritos en la cultura artística contemporánea, entre otras distinciones. 
tino en la década de los sesenta. A falta de otra documentación, podemos reconstruir la vinculación de Aguilera con el Partido Comunista en la clandestinidad a partir de los testimonios orales de varios miembros de la agrupación.

El pintor y miembro del Grupo Parpalló Doro Balaguer (Valencia, 1931) fue uno de los líderes del PCE en la Valencia de posguerra. Balaguer nos ayuda a datar con mayor exactitud la vinculación de Aguilera con el partido, ya que la colaboración de Balaguer con el Partido Comunista comenzó en 1956, y recuerda con claridad que cuando conoce a Aguilera con motivo de la formación del Grupo Parpalló ese mismo año, Vicente Aguilera no estaba comprometido con el partido. Sin embargo, tuvo evidencia de la relación de Aguilera con la organización comunista cuando, algunos años más tarde, llegó Antonio Palomares a Valencia -máximo dirigente del PCE en Valencia entre 1964 y 1979-, que fue exactamente en 1967. Doro Balaguer afirma que "el compromiso con la causa comunista de Vicente Aguilera es innegable, pero a la vez sabíamos que no estaba organizado dentro de la estructura de colaboración habitual, sino que ejercía una colaboración a la medida del desempeño de su trabajo"10.

Sobre el compromiso de Vicente Aguilera con el Partido Comunista nos da testimonio también Emèrit Bono i Martínez (Sagunto, 1940), Catedrático de Economía Aplicada de la Universitat de València y miembro del Comité Central del PCE durante la transición; él nos especifica que su relación con Aguilera se inició alrededor de 1966. Para Bono es difícil calibrar el grado de compromiso que adquirió Aguilera con el PCE durante la clandestinidad, pese a que asistió a varias reuniones que se convocaron en el domicilio del propio Bono. Según sus palabras, el principal papel que desarrolló Aguilera dentro del ámbito comunista fue el de servir de "puente, mediador o contacto entre los artistas del realismo social y crítico de mediados de los años sesenta y el PCE. A Aguilera podría atribuírsele el calificativo de 'intelectual orgánico' del PCE. Creo que Aguilera se planteaba su eventual militancia o acción política a través de su influencia en los ámbitos culturales, que ese era el modo de materializarse su compromiso"11. Recordemos que Aguilera en esos momentos escribe en la prensa local, es uno de los intelectuales de izquierdas de mayor prestigio internacional presente en la ciudad en esos años, y eso lo convertía en una persona influyente.

Por otro lado, Aguilera compartió tiempo, información y amistad con Antonio Palomares (El Robledo, Albacete, 1930-Valencia, 2007). Rosalía Sender Begué (Albalate de Cinca, Huesca, 1933), la esposa de Antonio Palomares, militante y destacada miembro de la dirección del PCEPV, nos confirma este dato, y proporciona un episodio clarificador. Justo unos días antes de la detención de Palomares en noviembre de 1968, Rosalía le había mostrado un número de la revista editada hasta el año anterior y que había dirigido Aguilera, Suma y sigue, entusiasmada por el talante del arte que proponía, y Palomares, señalando el nombre de Vicente Aguilera le comentó su interés para la causa. Aunque formaban matrimonio, "era una época en la que Antonio no daba datos de los militantes por la seguridad de ambas partes". Cuando Antonio Palomares es detenido días después, entre los mensajes que puede trasladarle a su esposa en las entrevistas que tiene con ella en prisión, en las que hablaban francés en argot para que los vigilantes no se enterasen, le pidió que le transmitiera a Aguilera que podía estar tranquilo, ya que no corría peligro ${ }^{12}$.

Otra forma de evaluar el compromiso político de Vicente Aguilera es a través de sus intervenciones en los encuentros y congresos profesionales fuera de nuestras fronteras a los que asistió, contempladas como ocasiones en las que optó por posicionarse y hacer visible la realidad española.

Tenemos varios ejemplos que así lo atestiguan, como es el caso de su participación de 1963 en la IV Biennale de San Marino y Veruccio. Según el expediente del Ministerio del Interior, Agui-

\footnotetext{
${ }^{10}$ Entrevista a Doro Balaguer el 20 de abril de 2012.

${ }^{11}$ Entrevista realizada a Emèrit Bono el 17 de mayo de 2012.

12 Entrevista realizada a Rosalía Sender Begué el 20 de abril de 2012.
} 
lera asistía a encuentros "de clara significación comunista como el congreso de AICA de 1963 en San Marino y Veruccio, en el que supuestamente estableció contactos con personas del mundo comunista e influyó para que se concediese la medalla de oro al pintor, también de ideología comunista, José García Ortega" ${ }^{13}$. Efectivamente, la IV Bienal de San Marino plasmaba su reflexión teórica en el Convegno de Rímini de 1963, organizado por un comité de críticos muy similar al de la bienal, y donde Aguilera tuvo un papel activo defendiendo la libertad de expresión y dedicando su escrito a Ibarrola y a Giménez Pericás, encarcelados en el penal de Burgos ${ }^{14}$.

Italia se convierte en altavoz de la cultura de oposición al franquismo desde finales de los años cincuenta, y en 1964, al hilo de la celebración de la Ventennale della Resistenza, este país albergó varias muestras de arte español ${ }^{15}$. Dentro de este marco se organiza la exposición de claros tintes antifranquistas titulada España libre como respuesta crítica a la celebración de los XXV Años de Paz que el régimen prepara, a través del ministro de Información y Turismo, Manuel Fraga. Esta muestra se llevó a cabo entre 1964 y 1965, por diferentes ciudades italianas, de manos de Giulio Carlo Argan y en la que participa activamente Vicente Aguilera junto a otros críticos e historiadores que también habían formado parte del jurado de la IV Bienal de San Marino y habían asistido al Convegno de Rímini. Vicente Aguilera será el introductor y difusor de las ideas de Giulio Carlo Argan (1909-1992) en España; con Argan se estableció una sintonía ideológica que les permitió trabajar de manera conjunta hasta el final de sus días. Ambos conciben el ejercicio de la crítica como parte de su compromiso ético, trabajando el pro de la relación entre la sociedad y el arte como base de su pensamiento crítico.

En el catálogo de la muestra, Vicente Aguilera escribirá un texto titulado "Sobre el significado de una cultura libre«, y lo dedicará de nuevo a Giménez Pericás e Ibarrola, quienes continuaban presos. El objetivo principal de la muestra era exhibir arte al margen del control del régimen, y Aguilera detallará en su texto "Es urgente rectificar la falsa impresión dada al mundo por las últimas exhibiciones burocráticamente patrocinadas, sea la exposición en la Tate Gallery (Londres, enero de 1962), sean las presentaciones en la VII Bienal de Sao Paulo (1963), en la XXXI o en la XXXII bienales de Venecia (1962 y 1964). Criterios absurdos y planteamientos incompetentes han estado echando por la borda el prestigio duramente ganado, ocasionando daño evidente a no pocos estimables artistas que incautamente aceptaron la vecindad con lamentables epígonos, con porcentajes suicidas de representación y sistemas presentativos absolutamente inadecuados que sólo

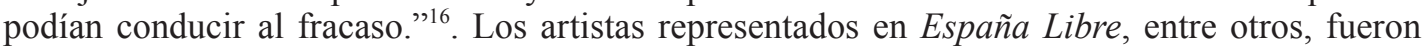
Eduardo Arroyo, Rafael Canogar, Estampa Popular, Manolo Millares, José Ortega, Antonio Saura, Eusebio Sempere y Antonio Tàpies.

Otra de las intervenciones internacionales de primer orden de Vicente Aguilera que nos ayudan a calibrar su compromiso ideológico con los ideales de izquierda -y que el régimen franquista persiguió- fue su participación del 10 al 15 de julio de 1965 en Helsinki en el denominado Congreso

${ }^{13}$ Informe de Ministerio de Información y Turismo - oficina de enlace, 7 de marzo de 1967, AGA, Cultura, caja 42/8820. También Carta de Carlos Areán a Vicente Aguilera Cerni, 17 de noviembre de 1967, archivo herederos Vicente Aguilera Cerni, Valencia (AHVAC). Sobre este evento y el posterior Congreso en Rímini ha publicado Barreiro, 2009b: 375-386.

${ }^{14}$ Para ahondar en estas reuniones ver Barreiro, 2009a: 308-310.

${ }_{15}$ Para conocer mejor el contexto italiano como plataforma de la cultura antifranquista recomendamos el texto de Núñez, 2004: 737-754.

${ }^{16}$ Aguilera, 1987: 146. El texto original fue escrito en italiano: "E' urgente rettificare l'errata impressione data al mondo dalle ultime mostre burocraticamente patrocinate, sia l'esposizione della Tate Gallery (Londra, gennaio 1962), sia le presenze alla VII Biennale di San Paolo 1963, nella XXXI o nella XXXII Biennale di Venezia (1962 e 1964). Criteri assurdi ed impostazioni incompetenti hanno gettato via il prestigio duramente guadagnato, causando danno evidente ad artisti, stimabili non poco, che incautamente accettarono la vicinanza con epigoni deplorevoli, con percentuali suicide di rappresentanza e sistemi di presentazione assolutamente inadeguati che potevano condurre soltanto al fallimento.” Aguilera, 1964. 
Mundial de la Paz. A esta gran convocatoria acudieron, entre otros, delegados de la talla de Pablo Neruda o Jean-Paul Sartre y Miguel Ángel Asturias.

Este organismo, el Consejo Mundial de la Paz, fue creado entre 1949 y 1950 como organización activista a favor de la paz, la igualdad, la defensa de los derechos humanos y el desarme general, y desde el inicio fue financiado casi en su totalidad por el Partido Comunista de la Unión Soviética (PCUS). Tras su organización se encontraba el departamento internacional del Comité Central del Partido Comunista soviético.

El Congreso Mundial de la Paz se propuso atraer a sus filas a intelectuales y pacifistas que sirvieran de atractivo mediático, que ayudasen a alzar sus voces a favor de los propósitos supuestamente pacifistas y a través de los cuales Moscú pretendía romper el equilibrio europeo camuflado de un movimiento en pro de la paz. Toda una campaña soviética en Occidente, que logró trazar una red de organizaciones en 135 países, dirigida en definitiva desde Moscú. La mayoría de los delegados que acudían a los congresos provenían de agrupaciones pro-comunistas, como en el caso de Picasso, Renato Guttuso o Diego Rivera ${ }^{17}$.

Vicente Aguilera Cerni pronunció un discurso como delegado español en dicho Consejo, que versó sobre la urgencia de proceder al desarme general y a la consecución de la paz, y calificó duramente, como habían hecho otros representantes, los bombardeos sobre Vietnam. Recalcó las palabras del profesor John Bernal sobre la voluntad planetaria para detener esa guerra. Además no quiso dejar de lado otros conflictos, como el que se estaba desarrollando desde el mes de abril de ese año en Santo Domingo, afectado por un golpe militar a un gobierno cuya modernidad hacía temer una ideología comunista latente, y que desembocó con la intervención de los Estados Unidos. Aguilera desea en su discurso para los dominicanos que puedan disponer de su destino sin interferencias extranjeras. También habló de la amenaza constante sobre Cuba, y tocó también otros focos candentes, como el rearme de Alemania Occidental, la intromisión en el Congo y otros lugares. Radio España Independiente -"Estación pirenaica", la clandestina emisora dirigida en un principio por Dolores Ibárruri- en la emisión de las 21 horas del día 21 de julio de 1965 se hizo eco de este discurso, calificándolo de muy relevante ${ }^{18}$.

Precisamente en 1965 Aguilera publicará en la revista Realidad, órgano cultural en el exilio del PCE. Realidad nació en 1963 y estaba dirigida por Jorge Semprún; tenía su sede en Roma, y entre su comité de redacción figuraban nombres como los del pintor José Ortega, Fernando Claudín, Francesc Vicens, Juan Gómez o Jesús Izcaray. Aguilera publicará un artículo que correspondía a la conferencia que había impartido en el encuentro de Rímini de ese año ${ }^{19}$.

En 1966 -año en que publica Panorama del nuevo arte español, y Ortega y D'Ors en la cultura artística española - tiene lugar el congreso de la Asociación Internacional de Críticos de Arte (AICA) en Praga, en el que Vicente Aguilera afirmó que la crítica de arte que se promovía en la España del régimen era apolítica, formalista y nacionalista. Según Paula Barreiro, Vicente Aguilera aprovecha su postura internacional desde la plataforma que le brinda la AICA para hacer activismo y que los demás componentes tomen una postura frente a la dictadura ${ }^{20}$.

Dentro del ámbito nacional Aguilera también se posicionó no sólo a través de sus textos y la gestión de grupos, revistas y exposiciones, sino también en el desarrollo de su profesión. Uno de los muchos episodios que nos demuestran hasta qué punto Aguilera sufría presiones por parte del gobierno franquista en el desarrollo de su labor debido a su oposición al régimen, es el que ha que-

\footnotetext{
${ }^{17}$ Para ampliar información sobre los congresos mundiales de la paz recomendamos el catálogo de Morris / Grunenberg, 2010.

${ }_{18}$ Informe de Ministerio de Información y Turismo - oficina de enlace, 21 de febrero de 1967, AGA, Cultura, caja 42/8820.

19 Aguilera, 1965: 7-32.

${ }^{20}$ Barreiro, 2009c: 116-119.
} 
dado reflejado en la correspondencia en torno a la revista Suma y sigue del arte contemporáneo, revista de gran relevancia por dar voz a los principales teóricos internacionales del momento, editada en Valencia entre 1962 y 1967 (Fig. 2).

En 1966 desde la Dirección General de Prensa del Ministerio de Información y Turismo que dirigía Manuel Fraga Iribarne, se le comunica la imposibilidad de ejercer la dirección de Suma y sigue -que inauguraba su segunda etapa- al no ostentar el carnet de periodista profesional, tal y como indicaba la nueva ley de prensa española de marzo de $1966 .{ }^{21}$

Aguilera decide no acatar en silencio la resolución y solicita protección internacional a la AICA con el objetivo de hacer valer en común los derechos profesionales de la crítica de arte; ${ }^{22}$ la reacción no se hace esperar, y una larga lista de personalidades del mundo artístico se alzan en apoyo suyo. Además, Jacques Lassaigne como Presidente de AICA, escribe al ministro Fraga para solicitar una excepción en el caso de Aguilera tras haber realizado una consulta legal que lo justifica. ${ }^{23} \mathrm{E}$ incluso desde dentro de la

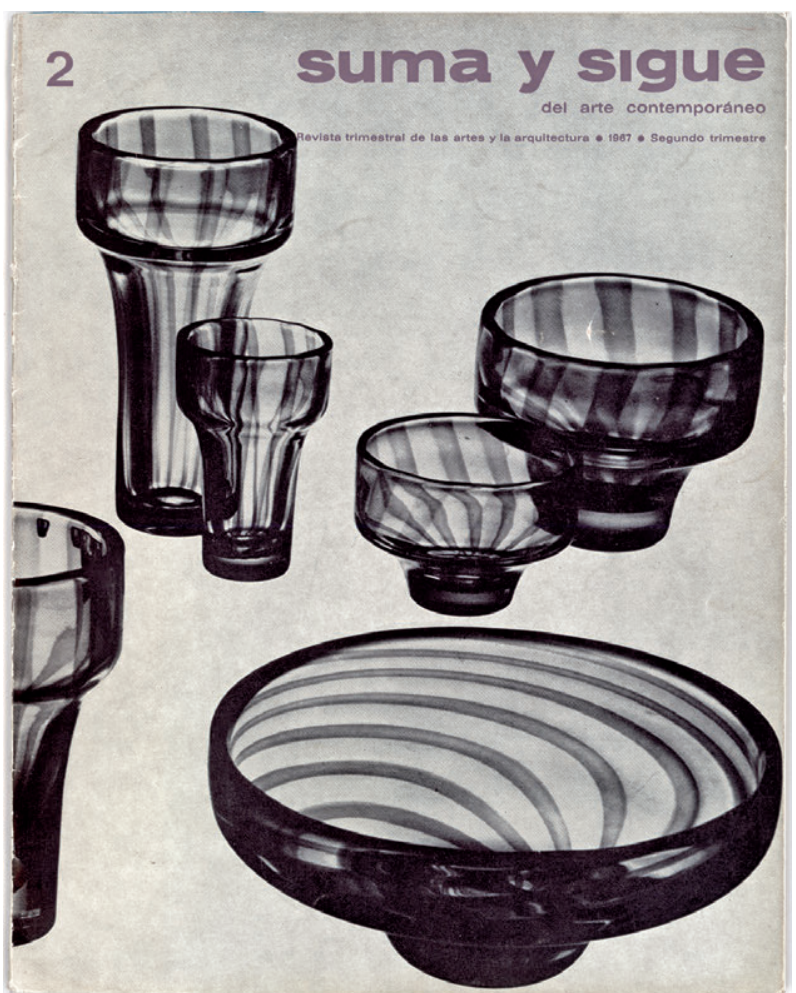

Fig. 2. Portada de la revista Suma y sigue, 2, segundo trimestre, 1967. Biblioteca Valenciana Nicolau Primitiu. administración, el propio Carlos Areán, como jefe de la sección de Artes Plásticas y Audiovisuales de la Dirección General de Información, también lo hizo a petición del artista Anzo.

En noviembre de 1967, Carlos Areán explica a Aguilera que obran en su poder unos datos ante los cuales no pueden hacer con él una excepción en el asunto mencionado. Estos datos abarcan detalladamente desde su filiación durante la Guerra Civil y la siguiente posguerra, su ideología, hasta su reciente intervención en Helsinki, demostrando que el régimen conocía de cerca cada uno de los gestos que llevaba a cabo Aguilera en pro de la denuncia de lo que estaba ocurriendo en España. ${ }^{24}$

Abatido, en diciembre de 1967, Aguilera comunica a José Huguet, editor de Suma y sigue, su determinación irrevocable de dimitir dadas las múltiples preocupaciones e inversión de trabajo que la revista le proporciona, y así poder dirigir sus esfuerzos a cumplir con los compromisos extranjeros que desean sus escritos y le otorgan el rango profesional que le reconocen ${ }^{25}$. Y efectivamente, hasta el último número publicado de la revista, Aguilera no aparece como director, sino como

${ }^{21}$ Carta de Vicente Aguilera Cerni a Jacques Lassaigne (Presidente de AICA), 2 de diciembre de 1966, AHVAC.

${ }^{22}$ Este tema lo ha estudiado Barreiro, 2014: 129-142.

${ }^{23}$ Se sumaron apoyos desde la Asociación Argentina de Críticos de arte, también Giulio Carlo Argan, Palma Bucarelli y un largo etcétera. La correspondencia entre ellos alude a que eran motivos políticos los que le impedían dirigir la revista. Entrevista realizada el 8 de mayo de 2012 a Mercedes Aguilera.

${ }^{24}$ Carta de Carlos Areán a Vicente Aguilera Cerni, fechada en Madrid el 17 de noviembre de 1967. AHVAC.

${ }^{25}$ Carta de Vicente Aguilera Cerni a José Huguet, fechada en Valencia el 25 de diciembre de 1967. AHVAC. 
asesor seguido de una larga lista de consejeros (entre los que se encontraban Giulio Carlo Argan, Umbro Apollonio, Jacques Lassaigne), y tras ellos un periodista director.

A raíz de este hecho, Aguilera informa a José Camón Aznar, presidente de la AECA, con el objeto de que -al margen de su caso personal-, el colectivo de críticos sea sabedor de esta norma y se movilice ante la posibilidad de cambiar el nuevo Estatuto de la Profesión Periodística, donde ya en otras disciplinas se exime de ser periodista para dirigir publicaciones técnicas y especializadas. En definitiva, reclama que la AECA se haga portavoz de los derechos de la crítica de arte española en vistas a este nuevo estatuto.

Otra de las acciones contestatarias en las que Aguilera se implicó a lo largo de estos años sesenta dentro del país consistió en sumarse a diversas protestas, firmando toda una serie de cartas colectivas contra diversas actividades del Estado español. Entre ellas figuran $-\mathrm{y}$ destacamos por ser de las primeras cartas colectivas que se envían firmadas por un conjunto de artistas y personas vinculadas a la cultura, y que demostró por primera vez una organización para movilizar una protesta-, la dirigida por un colectivo de intelectuales al Ministro de Información y Turismo en 1963 sobre los malos tratos ejercidos por la policía en Sama de Langreo, con motivo de las huelgas de Asturias. Como explica Mónica Núñez Laiseca, esta carta "representó en el área de cultura, un preámbulo de la lucha cada vez más abierta que iba a enfrentar, [...] a las autoridades franquistas y a los grupos de la oposición. [...] La implicación de la figura del intelectual -y, dentro de ella, del crítico o historiador del arte y del artista- en sondeos epistolares que pudieran generar una conciencia crítica frente a la dictadura, se demostró como un recurso de cierto alcance, tanto nacional como internacional, por lo que siguió utilizándose como revulsivo contra el sistema hasta los últimos días del franquismo"26.

También firmó una misiva dirigida al ministro Fraga en 1965 y remitida por el mismo colectivo de intelectuales, en la que se reivindicaba mayor libertad en diversos problemas nacionales. En 1966 se incorporó al colectivo que solicitaba flexibilidad frente al proyecto de la Nueva Ley de Prensa. Los sucesos acaecidos en Guantánamo también generaron una protesta escrita en 1966 por un grupo de intelectuales entre los que figuró Aguilera dirigida al Embajador de los Estados Unidos. Por último, la falta de información en la prensa local sobre los sucesos desarrollados en la Universidad de Valencia en 1967, fueron motivo de una misiva al Ministro de Información y Turismo en la que Vicente Aguilera también comprometió su firma. Recordemos que entre enero y febrero de 1967 se produce en dicha universidad el primer triunfo del movimiento democrático antifranquista de estudiantes universitarios, y tras la disolución del Sindicato Español Universitario (SEU), fracasa el intento del régimen de perpetuar su presencia en los claustros. La respuesta del gobierno se tradujo en un endurecimiento en la presión sobre el colectivo estudiantil. Como demuestran estos datos, Aguilera se posicionó claramente y así quedó reflejado en su expediente del Ministerio de Información y Turismo ${ }^{27}$.

Este expediente nos proporciona algunos datos más sobre la visión que el régimen tenía de Aguilera: se le califica como persona de pensamiento marxista, relacionado con individuos de izquierda, con disidentes filocomunistas y grupos separatistas valencianos en contacto con catalanistas. Se afirma que es miembro directivo del Ateneo Mercantil de Valencia, y que se relaciona con actividades comunistas y socialistas. Además, el expediente concluye con la frase hecha que lo etiqueta como "desafecto": se asegura que Aguilera es "asistente a tertulias liberales y separa-

\footnotetext{
${ }^{26}$ Según Mónica Núñez Laiseca, la primera carta de protesta contra el régimen firmada por intelectuales es el Documento dirigido por 102 intelectuales españoles a Fraga Iribarne, de septiembre de 1963 y por el mismo motivo que señalamos, y en el que no firmaba Aguilera. A esta carta Fraga respondió y se le volvió a enviar otra misiva reiterando la denuncia a la política de represión, y en esta segunda carta ya figura la firma de Aguilera. Núñez, 2006: 96-98.

${ }^{27}$ Informe de Ministerio de Información y Turismo - oficina de enlace, 7 de marzo de 1967, AGA, Cultura, caja $42 / 8820$.
} 
tistas que se celebran en el Ateneo Mercantil de Valencia, estando calificado como completo desafecto al actual sistema político español".

La invasión soviética de Checoslovaquia, condenada por otros estados comunistas, y el traumático desarrollo de la Primavera de Praga entre 1968 y 1969, provocarán el distanciamiento de Aguilera del PCE; serán estos los motivos que llevaron a Vicente Aguilera a emprender una profunda revisión de sus puntos de vista sobre el comunismo y a acercarse a las tesis del socialismo ${ }^{28}$.

Justo por estos años Aguilera está inmerso en la planificación del primer centro de arte contemporáneo de la Comunidad Valenciana, el Museo Popular de Arte Contemporáneo de Villafamés, - una pequeña localidad de la provincia de Castellón-, que sería una realidad en 1970. Aguilera configura un museo popular, construido a base de una cuidadosa selección de obras en préstamo, cesión o donación. Frente a la idea de museo exclusivo y elitista, Aguilera Cerni funda un museo del pueblo, democrático y renovable. El proyecto fue considerado un completo éxito, ya que activó la economía local y, consecuentemente, frenó la emigración juvenil de la localidad. Fue una empresa en la que Aguilera se implicó apasionadamente y a través de la cual logró realizar un importante cambio en un enclave, partiendo exclusivamente de un foco de cultura y arte ${ }^{29}$.

Una vez llega la tan esperada transición, Aguilera se incorpora como militante en el PSP. Pero ¿por qué se comprometió públicamente Vicente Aguilera con la opción del Partido Socialista Popular y no con la del PCE, su partido desde la juventud, una vez legalizada ya esta opción? Para Emèrit Bono vincularse con el PSP, el proyecto del profesor Tierno Galván, era socialmente más tolerable y más elegante ${ }^{30}$.

El Partido Socialista del Interior (PSI) es fundado por el profesor Enrique Tierno Galván (Madrid, 1918-1986) en 1968, y cambiará su nombre en 1974 por el de Partido Socialista Popular. Manuel Sánchez Ayuso (Murcia, 1941 - Valencia, 1982), catedrático de Política Económica y decano de la Facultad de Económicas de la Universidad de Valencia, fue miembro del PSI y alrededor suyo formará el PSP del País Valenciano, y lo dirigirá desde 1975 hasta 1978. Antes de la legalidad del PSP, Vicente Aguilera es elegido presidente del partido por sus compañeros, -aproximadamente a finales de 1975-, y ya tras la legalización en 1977, será reelegido en el primer y último congreso del PSP-PV celebrado en el mismo año.

El desarrollo del PSP-PV abarca de 1975 a 1978. Arranca en la ciudad de Valencia y en el entorno de Manuel Sánchez Ayuso: estudiantes y profesores del ámbito universitario, de la Facultad de Económicas en un primer momento. En el sector intelectual se sumó Vicente Aguilera; con él se afilian sus allegados, su hija Mercedes y su yerno el editor Fernando Torres, y sus amigos y colaboradores, como el crítico José Garnería, el artista José María Yturralde, el profesor Juan Ángel Blasco Carrascosa, entre otros muchos.

A las primeras elecciones democráticas, las de junio de 1977, se presentará el PSP formando parte de la coalición denominada Unidad Socialista, obteniendo a nivel nacional seis diputados, uno de ellos elegido por la provincia de Valencia. La integración del PSP-PV con el PSOE se producirá un año después, en junio de 1978, y en la comisión de trabajo estuvo implicado Aguilera junto a Víctor Fuentes, José Cabrera y Alfonso Goñi. Antes de la integración, el PSP-PV había alcanzado una cantidad modesta de militantes, alrededor de mil afiliados en la provincia de Valencia ${ }^{31}$ (Fig. 3).

Creemos necesario aproximarnos a los principales rasgos ideológicos del PSP con el que Aguilera se identificó, para entender su idiosincrasia; el programa del partido de Tierno Galván se declaraba republicano aunque admitía como paso previo otra forma de gobierno aceptada de manera democrática, propugnaba la separación entre Iglesia y Estado y concebía la cultura bajo el prisma

\footnotetext{
${ }^{28}$ Aguilera lo cuenta en la entrevista de Cal dir, realizada por Marí, 1978: 36-37.

${ }^{29}$ Para mas información sobre el proyecto consultar Blasco, 1995.

${ }^{30}$ Entrevista realizada el 17 de mayo de 2012.

${ }^{31}$ Sobre este tema recomendamos los libros de Sanz, 1988, y de Sanz, 1982.
} 
de la educación permanente, basada en la cooperación y no como arma de competición; abogaba por una concepción marxista de la evolución histórica al defender una sociedad sin clases. Promovía una abolición de la propiedad privada de los medios de producción, para lograr la autogestión de los trabajadores, en los medios agrarios, industrial y de servicios; además, se definía como un partido internacionalista que creía en la unidad de los trabajadores sin sujeción de fronteras y en defensa de la unidad europea ${ }^{32}$.

Además de las tareas desempeñadas por Aguilera en el partido, -pensar estrategias para ampliar la militancia, discutir la toma de posiciones respecto a temas de actualidad y tener presencia en los debates públicos-, podemos sopesar la esencia de su aportación al PSP a través del análisis del programa cultural que redactó para el ámbito valenciano, de cara a las elecciones de 1977. Junto a Juan Ángel Blasco Carrascosa tradujo y adaptó al caso valenciano los programas del partido socialista francés y del partido comunista italiano ${ }^{33}$. En dicha propuesta Aguilera concebía la cultura como un factor esencial para la consecución de una sociedad mejor, en la que "cada individuo pueda lograr la plenitud

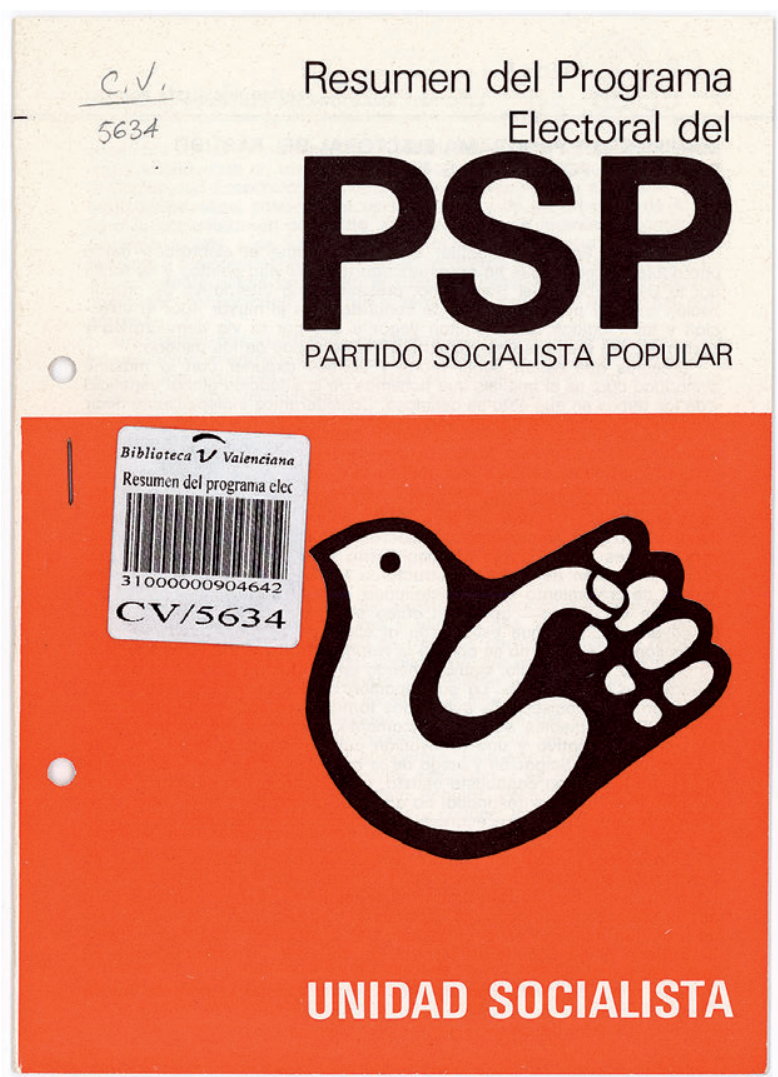

Fig. 3. Resumen del programa electoral del PSP, Partido Socialista Popular-Unidad Socialista, 1977. Biblioteca Valenciana Nicolau Primitiu. intelectual, el equilibrio espiritual y la elevación moral." Proponía también planificar desde las pequeñas comunidades rurales y barrios, creando "centros comunitarios con el más amplio equipamiento en todas las comunidades y barriadas, propiciando su gestión democrática, su condición de centros vivos de convivencia comunitaria a la vez que enclaves básicos de la actividad cultural y artística entendida de modo interdisciplinar." Las medidas recogidas en el programa cultural intentaban paliar las distancias socioeconómicas entre los ámbitos rurales y los urbanos. Ya hablaba Aguilera de la lucha por una jornada laboral que "permita al trabajador su cultivo intelectual y el desarrollo de su personalidad así como su esparcimiento". Este programa apoyaba las medidas fiscales en pro de la desprivatización -mediante estímulos y no de manera restrictiva- de los bienes culturales a favor de la mejora cultural comunitaria. También contemplaba el programa la socialización del suelo urbano y urbanizable para hacer una política integradora del hombre con el entorno. ${ }^{34}$

${ }^{32}$ PSP - Partido Socialista Popular del País Valenciano. Programa, Valencia, PSP-PV, 1977.

${ }_{33}$ Entrevista a Juan Ángel Blasco Carrascosa realizada el 12 de abril de 2012.

34 VVAA., 1977: 89, 90 y 91. En un momento político con tantas opciones posibles, Aguilera quiso llevar a cabo una labor clarificadora, y entendió que el museo de Villafamés podía implicarse publicando en un pequeño volumen las opciones culturales de Alianza Popular, Partit Comunista del PV, Partido Demócrata Liberal, Partido Laborista, Partido Popular, PSOE del PV, PSP-PV, Partit Socialista del PV y Unió Democrática del PV. 
Víctor Fuentes Prósper, profesor de la Facultad de Económicas, destacado primer y único secretario ejecutivo del PSP-PV, afirma que la idea de revolución cultural de Tierno Galván había calado hondamente en Vicente Aguilera Cerni ${ }^{35}$. Tierno abogaba por la necesidad de llevar a cabo una revolución cultural no violenta, que debía empezar en la escuela, extenderse por la familia, continuar en la universidad y en la convivencia. Se trataba en definitiva de una revolución cultural que orientaría una revolución social. La cultura del capitalismo era caduca, afirmaba Tierno, y por lo tanto Occidente necesitaba una nueva escala de valores, basada en una revolución cultural, que provocaría que el hombre tomase progresiva conciencia ante los problemas que le acucian, y dejaría de contemplar solamente el problema de su salario. Para Tierno, la revolución cultural debía caminar de forma paralela a la revolución política.

Las palabras del viejo profesor son vehementes al respecto: "La idea de separación entre cultura y política es en sí misma perversa. Destroza el equilibrio de la sociedad y oculta la conciencia de lo universal, que son imprescindibles para que una comunidad no se corrompa y sus miembros no se dejen coger por la indiferencia y la despreocupación respecto de todo lo que no sean ellos mismos. Nuestra obligación como políticos [...] sería volver a enlazar cultura y política" ${ }^{36}$.

Al hilo de estas ideas retomamos la figura de Argan. Ya hemos señalado que la coincidencia ideológica con Giulio Carlo Argan les permite ser colaboradores durante décadas, ya que ambos creen en la responsabilidad del arte para comprometerse con su tiempo. En 1976 Argan también se implica políticamente y es elegido alcalde de Roma por el Partido Comunista Italiano, aunque en calidad de independiente. Aguilera, que considera a Argan su maestro, publicará sobre este hecho y no creemos desaventurado definir al Aguilera político a través de sus palabras sobre Argan, en las que podemos encontrar rasgos de su propia ideología: "El socialismo de Argan, siendo marxista, es antidogmático y creativo. Es un socialismo de izquierdas. En el fondo, su dimensión cultural le instala en la dialéctica histórica de un socialismo que no puede fosilizarse, que ha de ser -con exigente rigor autocrítico- la conciencia viva de una historia donde, asumiendo las enseñanzas del pasado, se interviene en el presente para configurar un futuro mejor" ${ }^{37}$. Para Aguilera existe un claro paralelismo entre el trabajo que tiene que desempeñar Argan en Italia y el caso español: se ha de atacar la especulación inmobiliaria y gestionar y conservar un inmenso patrimonio para que sea entendido como fuente de riqueza de la ciudad. Argan declaró que no se podía ser historiador sin tener intención política, y entendía el socialismo como portador de ideas que convierten a la cultura en algo moderno y actual, y que los fenómenos sociales tienen sobre la génesis del arte una importancia fundamental. Todos estos rasgos los encontramos también en la figura del Aguilera comprometido con la transición española.

A finales de la década de los setenta, Vicente Aguilera comienza a fraguar la idea de formar una asociación que respalde el oficio desempeñado por los críticos de arte del ámbito valenciano, y en 1980 finalmente se constituye la AVCA (Asociación Valenciana de Críticos de Arte). Aguilera desempeñará las labores de presidente desde 1980 a 1987, cargo que dejó de ejercer para poder asumir la presidencia de la Asociación Española de Críticos de Arte ${ }^{38}$. Y poco antes, en 1979, había puesto en marcha su último proyecto de publicación periódica, la revista Cimal, subtitulada como Cuadernos de cultura artística durante su primera etapa y como Arte internacional en la segunda, que se editaría hasta 2003. Su espíritu emprendedor y activista era, como vemos, incansable.

\footnotetext{
${ }^{35}$ Entrevista a Víctor Fuentes Prósper realizada el 9 de mayo de 2012.

36 Tierno, 1981: 83.

37 Aguilera, 1976: 67-69.

${ }^{38}$ Para profundizar en esta etapa recomendamos la lectura de De la Calle, 2012: 133-172.
} 
Su compromiso no acabó aquí: sabemos que en 1981 Ciprià Císcar, quien ocupa el cargo de conseller de Cultura en el gobierno socialista, nombra a Vicente Aguilera asesor de esa conselleria. Y hasta el último de sus días, Vicente Aguilera Cerni, mantendría su afiliación al PSOE. ${ }^{39}$

En 1985 la Generalitat Valenciana crea una entidad asesora en materia de cultura, denominada Consell Valencià de Cultura (CVC), con la finalidad de promover un foro de discusión sobre temas complejos, al margen del debate partidario. La presidencia fue ocupada por el poeta Juan Gil-Albert, y Aguilera, que desarrollaba la función de consejero, pasó a ocupar al poco tiempo el cargo de vicepresidente de la institución. En 1994 Vicente Aguilera Cerni asumió la presidencia del CVC, hasta 1996, año en que se encargaría al científico Santiago Grisolía. Entre las tareas que desempeñó Aguilera, cabe destacar la recuperación de piezas de autores valencianos enviadas al Pabellón español de la Exposición Internacional de París de 1937. ${ }^{40}$

Aguilera también plasmará su compromiso en sus publicaciones. A lo largo de los años sus textos apostarán por un arte integrado en las raíces de la sociedad, no elitista; exigirá, tanto al artista como al crítico, responsabilidad en su trabajo dadas las inmediatas consecuencias sociales que se derivan del mismo, y en definitiva defenderá la activación de un arte no acomodado, sino implicado con la sociedad que lo produce.

Podemos concluir que Vicente Aguilera Cerni ejerció un compromiso político constante a lo largo de su vida, y que sus convicciones lo guiaron tanto en su proceder como en sus escritos por varias sendas, progresistas todas ellas, y en cuya base estaba la firme creencia de que la cultura y el arte, en libertad, eran elementos indispensables para la construcción de un hombre completo.

\section{BIBLIOGRAFÍA}

Aguilera Cerni, Vicente (1964): "Sul significato di una cultura libera". En AA.VV.: España libre. Esposizione d'arte spagnola contemporánea. Rimini: Palazzo dell’Arengo, s.p.

Aguilera Cerni, Vicente (1965): "Sobre las posibilidades de comunicación de las artes visivas en las sociedades actuales". En: Aguilera Cerni, Vicente (1973): Posibilidad e imposibilidad del arte. Valencia: Fernando Torres.

Aguilera Cerni, Vicente (1976): "Giulio Carlo Argan, alcalde de Roma: la cultura artística al gobierno de la ciudad". En: Guadalimar, 17 (10 de noviembre), pp. 67-69.

Aguilera Cerni, Vicente (1987): "Sobre el significado de una cultura libre". En: Aguilera Cerni, Vicente (1987): Textos, pretextos y notas. Textos escogidos, 1953-1987. Valencia: Ayuntamiento de Valencia, pp. 143-146.

Aguilera Cerni, Vicente (2010): Historia y presagio. Poemas 1941, Rubielos de Mora (Teruel): Museo Salvador Victoria; Madrid: Asociación Española de Críticos de Arte.

Barreiro López, Paula (2009a): La abstracción geométrica en España (1957-1969). Madrid: CSIC, 2009.

Barreiro López, Paula (2009b): "La Biennale de San Marino et le Congrès de Rímini de 1963: Argan, Restany et Aguilera Cerni vers un art engagé”. En Leeman, Richard (dir.) (2009) : Le démi-siècle de Pierre Restany. París: INHA, pp. 375-386.

Barreiro López, Paula (2009c): "Nulla aesthetica sine ethica: le critique d'art espagnol Vicente Aguilera Cerni”. En: Critique d'Art, 34 (automne), pp. 116-119.

Barreiro López, Paula (2014): "Redes críticas: encuentros e intercambios de España a la Europa de la Guerra Fría". En: Cabañas Bravo, Miguel y Rincón García, Wifredo (eds.) (2014): Las redes del arte español desde 1900. Madrid: CSIC, pp. 129-142.

Bernárdez Sanchís, Carmen (2010): “Ímpetu y sueño del arte norteamericano en los escritos de Aguilera Cerni”. En: Revista Complutense de Historia de América, 36, Madrid, pp. 127-149.

Blasco Carrascosa, Juan Ángel (1995): Museo popular de arte contemporáneo de Vilafamés, catálogo-guía. Valencia: Consell Valencià de Cultura.

\footnotetext{
${ }^{39}$ Entrevista a Mercedes Aguilera realizada el 8 de mayo de 2012.

${ }^{40}$ El Consell Valencià de Cultura acaba de publicar la biografía de Vicente Aguilera donde se recopila este hecho, de la mano de Blasco, 2011.
} 
Blasco Carrascosa, Juan Ángel (2011): Vicente Aguilera Cerni. Valencia: Consell Valencià de Cultura.

De la Calle, Román (2012): "Vicente Aguilera Cerni (1920-2005) y la Asociación Valenciana de críticos de arte (AVCA) (1980-2010). 30 años de trayecto”. En: De la Calle, Román (2012): A propósito de la crítica de arte. Teoría y práctica. Cultura y política. Valencia: Universitat de València, pp. 133-172.

Delgado Gómez-Escalonilla, Lorenzo (2009): "La maquinaria de la persuasión. Política informativa y cultural de Estados Unidos hacia España”, En: Ayer, 75, pp. 97-132.

Delgado Gómez-Escalonilla, Lorenzo (2012): "Objetivo: atraer a las élites. Los líderes de la vida pública y la política exterior norteamericana en España”. En: Niño, Antonio/ Montero, José Antonio (eds.) (2012): Guerra Fría y Propaganda. Estados Unidos y su cruzada cultural en Europa y América Latina. Madrid: Biblioteca Nueva.

León Aguinaga, Pablo (2009): “Los canales de la propaganda norteamericana en España, 1945-1960”. En: Ayer, 75, pp. $133-158$

Mancebo, Ma Fernanda (1988): La Universidad de Valencia en guerra. La FUE (1936-1939). Valencia: Ajuntament, Universitat.

Marí, Rafa (1978): “Amics i coneguts: Vicente Aguilera Cerni” [entrevista]. En: Cal dir, 72, 20 de noviembre, Valencia, pp. 36-37.

Morris, Lynda/ Grunenberg, Christoph (eds.) (2010): Picasso: peace and freedom. London: Tate Publishing.

Núñez Laiseca, Mónica (2004): "Los caminos del arte español en Italia: una evocación de la resistencia”. En: VVAA. (2004): Congreso de Caminería hispánica (actas). Madrid: Ministerio de Fomento, pp. 737-754.

Núñez Laiseca, Mónica (2006): Arte y política en la España del desarrollismo (1962-1968). Madrid: CSIC.

Sanz Díaz, Benito (1988): Los socialistas en el País Valenciano (1939-1978). Valencia: Alfons el Magnànim - Institució valenciana d'estudis i investigació.

Sanz, Jesús (1982): La cara secreta de la política valenciana. De la Predemocracia al Estatuto de Benicàssim. Valencia: Fernando Torres.

Tierno Galván, Enrique (1981): Cabos sueltos. Barcelona: Bruguera.

VVAA. (1977) Cultura y política. Encuentros de Villafamés. Valencia: Fernando Torres.

Fecha de recepción: 24-III-2015

Fecha de aceptación: 25-VI-2015 